\title{
Low $x$ Physics at the LHeC
}

\author{
Paul Newman \\ School of Physics \& Astronomy, University of Birmingham, B15 2TT, UK
}

\begin{abstract}
A first sketch is developed of the wealth of opportunity for exploring low $x$ physics afforded by the proposed LHeC electron-nucleon (or nucleus) collider.
\end{abstract}

\section{Introduction}

This contribution [1] is concerned with the LHeC [2], a proposed future deep-inelastic scattering (DIS) facility at CERN, where protons or heavy ions from an existing $7 \mathrm{TeV}$ LHC storage ring collide with electrons from a newly built machine (nominally $70 \mathrm{GeV}$ ) in the same tunnel, simultaneously with proton-proton or heavy ion collisions at the existing LHC experiments. The expected luminosity of $10^{33} \mathrm{~cm}^{-2} \mathrm{~s}^{-1}$ is two orders of magnitude larger than has been achieved at HERA and the centre of mass energy of $1.4 \mathrm{TeV}$ is five times larger than that at HERA. Here, a first exploration is made of the rich physics potential resulting from the extension by typically a factor of 30 towards lower $x$ at the LHeC compared with HERA (figure 1). Complementary contributions on sensitivity to parton densities and to new physics are covered in [3] and [4], respectively.
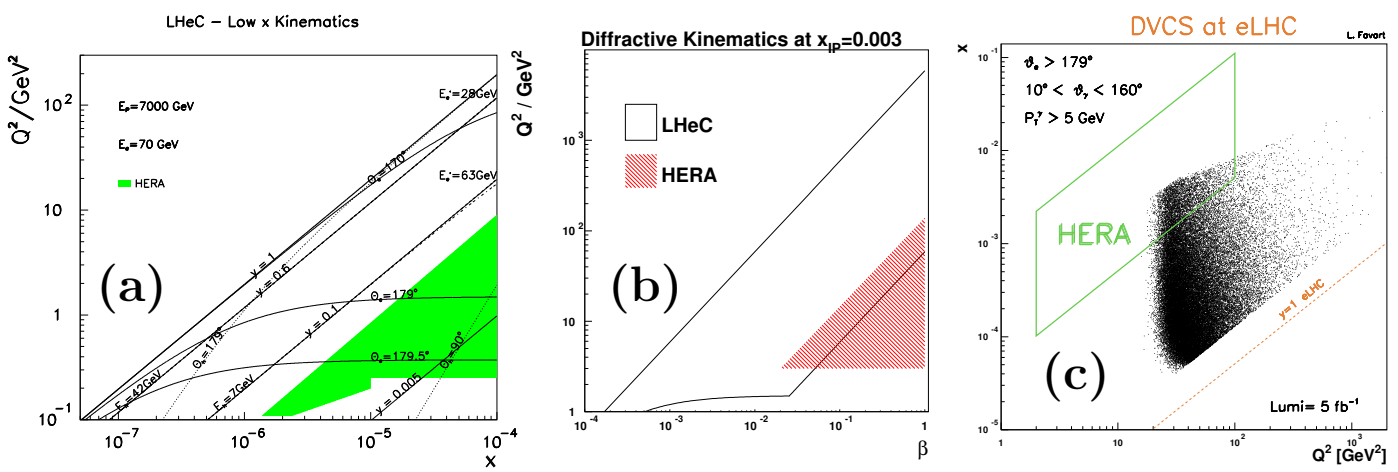

Figure 1: (a) The low $x$ corner of the $x-Q^{2}$ kinematic plane at HERA and the LHeC. (b) The $\beta-Q^{2}$ kinematic plane for diffractive DIS at HERA and the LHeC for an example $x_{\mathbb{P}}=0.003$. (c) Simulated DVCS events at the LHeC, illustrated in the $x-Q^{2}$ kinematic plane and compared with the coverage of HERA [5].

The most enduring legacy of HERA is likely to be its pioneering contribution to low $x$ physics. Most notable among the many discoveries are the strong rise of the proton quark $\left(\sim F_{2}\right)$ and gluon $\left(\sim \partial F_{2} / \partial \ln Q^{2}\right)$ densities with decreasing $x$. The phenomenological consequences of the resulting high density, low coupling limit of QCD have now been explored in considerable detail. New processes such as diffractive DIS $(e p \rightarrow e X p)$ and Deeply Virtual Compton Scattering (DVCS, $e p \rightarrow e \gamma p$ ) have been investigated for the first time. Yet many things remain rather unclear, in particular how and at which $x$ values the increasing gluon density is tamed, as required to satisfy unitarity.

A significant problem in understanding low $x$ physics at HERA is the kinematic correlation between the low $x$ and low $Q^{2}$ regions (figure 1a), such that any novel low $x$ parton 
dynamics are usually entangled with effects due to the low $Q^{2}$ breakdown of perturbative QCD and the transition to the region in which quarks and gluons cease to be appropriate degrees of freedom. In order to access the very low $x$ region where non-linear effects may be present [6], $Q^{2}$ values well below typical hadronic mass scales are therefore required at HERA, precluding a clear partonic interpretation. In contrast, it is likely to be possible ${ }^{\text {a }}$ to observe non-linear evolution effects at the $\mathrm{LHeC}$ at $Q^{2}$ values for which an unambiguous partonic interpretation can be made (see section 3 ).

\section{Low $x$ Detector Considerations}

The lower limit in accessible $Q^{2}$, and hence in $x$, is determined principally by the LHeC detector coverage at low electron scattering angles. The proposed beam focusing magnets [2], used to maximise the luminosity for searches and other high momentum transfer studies [4], would prevent instrumentation within $10^{\circ}$ of the beam-pipe, resulting in little acceptance for $Q^{2}<100 \mathrm{GeV}^{2}$ or $x<5.10^{-5}$. Without these focusing magnets ${ }^{\mathrm{b}}$ the acceptance could extend within $1^{\circ}$ of the beam-pipe, giving access down to $x=5.10^{-7}$ at $Q^{2}=1 \mathrm{GeV}^{2}$, whilst still collecting $1 \mathrm{fb}^{-1}$ of data per year.

With the LHeC beam energies, the scattered quark direction generally lies close to central rapidity for the newly accessed lowest $x$ range. However, the LHeC hadronic final state is boosted in the forward (outgoing proton beam) direction more strongly than is the case at HERA for the same $x$ values. Placing detector components within $1^{\circ}$ of the beam pipe is thus also beneficial in the forward direction. Accessing the hadronic final state over a wide rapidity range allows the inclusion of hadronic variables in the kinematic reconstruction and will be important for studies of the initial state parton cascade through forward jets, to distinguish between different parton emission models [8]. The large forward boost also has implications for studies of diffraction. A diffractive event with fractional proton longitudinal momentum loss $x_{I P}=0.01$ typically deposits its most forward hadronic final state particles at pseudorapidities $\eta_{\max } \sim 5$ at the LHeC, compared with $\eta_{\max } \sim 3$ at HERA. Very forward instrumentation will therefore be required if large rapidity gap selections are to be made. Forward proton and neutron detectors should also be an integral part of any detector design.

\section{Example Low $x$ Case Studies}

- Inclusive Cross Sections and Parton Saturation: It is expected that parton saturation effects could be conclusively established at the LHeC through the observation of deviations from expectations for one or more observable in the framework of perturbative QCD. However, in such studies at HERA, models based on colour dipole scattering $[6,9,10]$ have been used in order to access the necessary very low $Q^{2}$ values.

In one example dipole study of HERA data [9], the inclusive structure function $F_{2}\left(x, Q^{2}\right)$ is subjected to fits in which the dipole cross section either does not exhibit saturation properties, or saturates as expected in two rather different models [9, 10]. All three dipole fits are able to describe the HERA data adequately in the perturbative region $Q^{2} \geq 2 \mathrm{GeV}^{2}$,

\footnotetext{
${ }^{\text {a}}$ Quantitatively, in the language of geometric scaling [7], the observed change in behaviour of the scaling curve for HERA data around $\tau=Q^{2} R_{0}^{2}(x)=1, R_{0}$ being the 'saturation radius' [6], is often considered as evidence for saturation. At HERA, all data with $\tau<1$ correspond to $Q^{2} \ll 1 \mathrm{GeV}^{2}$. In contrast, at the LHeC, $\tau \simeq 0.15(0.4)$ is reached for $Q^{2}=1(2) \mathrm{GeV}^{2}$.

${ }^{b}$ Ideally the $\mathrm{LHeC}$ design will allow switches between configurations with and without these magnets.
} 
whereas a clear preference for the models containing saturation effects becomes evident when data from the range $0.045<Q^{2}<1 \mathrm{GeV}^{2}$ are added [9]. Due to the non-perturbative nature of this kinematic region, there is no clear interpretation in terms of parton recombination effects. Similar conclusions are drawn when the same dipole cross sections are applied to various final state observables [11].

Figure 2 shows an extrapolation of the three dipole models [9] into the LHeC kinematic range at an example $Q^{2}=10 \mathrm{GeV}^{2}$. The extrapolations are compared with a simulated $\mathrm{LHeC}$ measurement with $1 \mathrm{fb}^{-1}$, where statistical errors are negligible and reasonable estimates of systematic errors are at the $1-3 \%$ level [3]. The $\mathrm{LHeC}$ data clearly distinguish between the extrapolated fits to HERA data without resorting to a region where perturbative methods are inapplicable. It remains to be shown whether this continues to be the case when the LHeC data are also included in the fits.

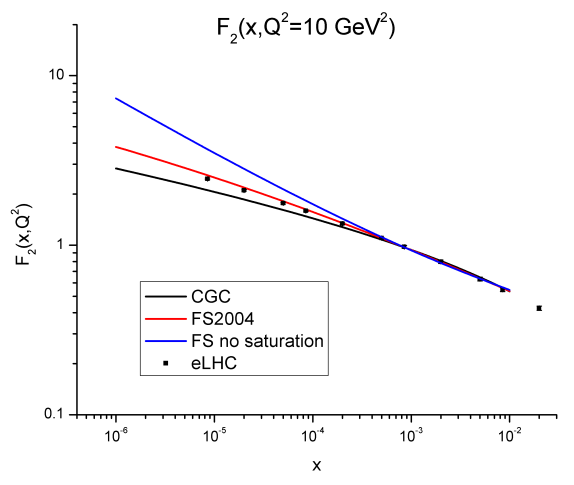

- Diffractive DIS: Statistical uncertainties should be insignificant for the measurement of a diffractive DIS cross section with $1 \mathrm{fb}^{-1}$ at the LHeC. Systematic errors are estimated to be in the region of $5-10 \%$,

Figure 2: Example results from fits to HERA $F_{2}$ data with $10^{-4} \lesssim x<10^{-2}$ using three different dipole models. The curves are extrapolated to lower $x$ values [12] and compared with simulated LHeC data. depending strongly on the design of the forward region of the detector. At an example $Q^{2}=10 \mathrm{GeV}^{2}, x_{P}$ values below $10^{-5}$ are accessible, allowing a very clean separation of the diffractive exchange from sub-leading contributions. The $\beta=x / x_{\mathbb{P}}$ and $Q^{2}$ kinematic plane at HERA and the LHeC is illustrated in figure $1 \mathrm{~b}$, for an example $x_{\mathbb{P}}=0.003$. Accessing higher $Q^{2}$ at fixed $\beta$ and $x_{P}$ will test the factorisation properties of diffraction [13] in detail and will allow more precise constraints on diffractive parton distribution functions (DPDFs), including sensitivity to their flavour decomposition through $W$ and $Z$ exchange contributions. The low $\beta$ region of the DPDFs will be investigated for the first time.

The tests of diffractive factorisation carried out at HERA have largely involved applying DPDFs to the prediction of diffractive jet or charm cross sections, which are sensitive to the gluon density through the boson gluon fusion process $\gamma^{*} g \rightarrow q \bar{q}$. This procedure is kinematically restricted to $p_{\perp}<M_{\mathrm{X}} / 2 \lesssim 15 \mathrm{GeV}$, such that the large scale uncertainties in theoretical predictions now limit the precision of comparisons. The more favourable kinematics at the $\mathrm{LHeC}$ allow $M_{\mathrm{X}}$ values up to several hundred $\mathrm{GeV}$ to be accessed for reasonably small values of $x_{I P}$. This will improve the precision with which jets and charm can be used for factorisation tests and will also open up completely new topics such as diffractive beauty, $W$ and $Z$ production, or even the exclusive production of new $1^{--}$states.

- Deeply Virtual Compton Scattering As at HERA, measurements of DVCS could be made at the LHeC through the inclusive selection of $e p \rightarrow e p \gamma$ events and the statistical 
subtraction of the Bethe-Heitler background. A first simulation [5] assuming that final state photons with $p_{\perp}>5 \mathrm{GeV}$ can be efficiently selected, leads to the simulated data shown in figure 1c. With $10 \mathrm{fb}^{-1}$, a measurement at $Q^{2}=30 \mathrm{GeV}^{2}$ could be performed in seven bins of $W$ between $150 \mathrm{GeV}$ and $750 \mathrm{GeV}$, with $1-4 \%$ statistical precision. The LHeC data are clearly complementary in coverage to their HERA counterparts and will thus give fresh information on Generalised Parton Densities.

\section{Concluding Remarks}

The natural next step in the further pursuit of low $x$ physics with unpolarised hadrons is an extension of DIS studies to higher energy, equivalently, lower $x$. The LHeC provides a credible proposal for how this might be achieved in the framework of the LHC, whose unprecedented energy and luminosity are likely to make it the dominant high energy facility for the foreseeable future.

The studies presented here involve only a small fraction of the low $x$ physics topics which could be addressed with the LHeC. For example, no studies have yet been performed of jet, charm, beauty, prompt photon or vectotr meson cross sections, $F_{L}$ measurements or photon structure sensitivity. The possibilities with the amplified low $x$ parton densities available when the proton beam is replaced by lead ions also remains largely unexplored to date, but must surely have a major impact on nuclear parton density, nuclear shadowing and low $x$ saturation physics. There is thus vast scope for further study in order to fully evaluate the physics possibilities of an LHeC.

\section{Acknowledgements}

Thanks to E. Avsar, J. Dainton, L. Favart, J. Forshaw, M. Klein, L. Lonnblad, A. Mehta, E. Perez, G. Shaw and F. Willeke for their highly valued contributions to this presentation.

\section{References}

[1] Slides: http://indico. cern. ch/contributionDisplay $\cdot$ py? contribId=268\&sessionId=10\&conf Id=9499

[2] J. Dainton et al., JINST 1 (2006) P10001; J. Dainton, these proceedings.

[3] M. Klein, these proceedings.

[4] E. Perez, these proceedings.

[5] L. Favart, private communication.

[6] K. Golec-Biernat, M. Wüsthoff, Phys. Rev. D59 (1999) 014017.

[7] A. Stasto, K. Golec-Biernat, J. Kwiecinski, Phys.Rev.Lett. 86 (2001) 596.

[8] 'The THERA book: ep scattering at $\sqrt{s}=1$ TeV', DESY 01-123F, eds. U. Katz et al.

[9] J. Forshaw, G. Shaw, JHEP 0412 (2004) 052.

[10] E. Iancu, K. Itakura, S. Munier, Phys. Lett. B590 (2004) 199;

H. Kowalski, L. Motyka, G. Watt, Phys.Rev. D74 (2006) 074016.

[11] J. Forshaw, R. Sandapen, G. Shaw, JHEP 0611 (2006) 025.

[12] J. Forshaw, private communication.

[13] For a HERA investigation, see e.g. H1 Collbaoration, Eur. Phys. J. C48 (2006) 715, ibid 749. 